\title{
BMJ Open Randomised controlled trial into the role of ramipril in fibrosis reduction in rheumatic heart disease: the RamiRHeD trial protocol
}

\author{
Ade Meidian Ambari (D) ,1,2 Budhi Setianto, ${ }^{1,2}$ Anwar Santoso, ${ }^{1,2}$ Basuni Radi, ${ }^{1,2}$ \\ Bambang Dwiputra, ${ }^{1,2}$ Eliana Susilowati, ${ }^{3}$ Fadilla Tulrahmi, ${ }^{3}$ Annemiek Wind, ${ }^{4}$ \\ Maarten Jan Maria Cramer, ${ }^{4}$ Pieter Doevendans ${ }^{4,5}$
}

To cite: Ambari AM, Setianto B, Santoso A, et al. Randomised controlled trial into the role of ramipril in fibrosis reduction in rheumatic heart disease: the RamiRHeD trial protocol. BMJ Open 2021;11:e048016. doi:10.1136/ bmjopen-2020-048016

- Prepublication history for this paper is available online. To view these files, please visit the journal online (http://dx.doi org/10.1136/bmjopen-2020048016).

Received 17 December 2020 Accepted 25 August 2021

Check for updates

(C) Author(s) (or their employer(s)) 2021. Re-use permitted under CC BY-NC. No commercial re-use. See rights and permissions. Published by BMJ.

For numbered affiliations see end of article.

Correspondence to Mr Ade Meidian Ambari; dr_ade_meidian@yahoo.co.id

\section{ABSTRACT}

Introduction Rheumatic heart disease (RHD) is a major burden in developing countries and accounts for $80 \%$ of all people living with the disease, where it causes most cardiovascular morbidity and mortality in children and young adults. Chronic inflammation and fibrosis of heart valve tissue due to chronic inflammation in RHD will cause calcification and thickening of the impacted heart valves, especially the mitral valve. This fibrogenesis is enhanced by the production of angiotensin II by increased transforming growth factor $\beta$ expression and later by the binding of interleukin-33, which is known to have antihypertrophic and antifibrotic effects, to soluble sST2. sST2 binding to this non-natural ligand worsens fibrosis. Therefore, we hypothesise that ACE inhibitors (ACEIs) would improve rheumatic mitral valve stenosis.

Methods and analysis This is a single-centre, doubleblind, placebo-controlled, randomised clinical trial with a pre-post test design. Patients with rheumatic mitral stenosis and valve dysfunction will be planned for cardiac valve replacement operation and will be given ramipril $5 \mathrm{mg}$ or placebo for a minimum of 12 weeks before the surgery. The expression of ST2 in the mitral valve is considered to be representative of cardiac fibrosis. Mitral valve tissue will be stained by immunohistochemistry to ST2. Plasma ST2 will be measured by ELISA. This study is conducted in the Department of Cardiology and Vascular Medicine, Universitas Indonesia, National Cardiac Center Harapan Kita Hospital, Jakarta, Indonesia, starting on 27 June 2019.

Ethics and dissemination The performance and dissemination of this study were approved by the ethics committee of National Cardiovascular Center Harapan Kita with ethical code LB.02.01/VII/286/KEP.009/2018.

Trial registration number NCT03991910.

\section{INTRODUCTION}

Rheumatic heart disease (RHD) is a serious health problem in developing nations, where it affects $80 \%$ of the population and accounts for the majority of cardiovascular morbidity and mortality in children and young adults. RHD affects more than 15.6 million
Strengths and limitations of this study

- A novel study that analysed the ST2 expression in mitral valves in patients with rheumatic heart.

- This study proposed novel and affordable treatment targeting the rheumatic heart valve fibrosis reduction.

- This research will help low-to-middle-income countries treat rheumatic heart disease more economically.

- Flexible schedule of mitral valve surgery causes different time range of the intervention for each patient.

- No standard healthy control of the non-fibrotic valve, based on ethical consideration.

individuals worldwide, with 233000 people dying prematurely each year. ${ }^{1}$ In the past 5 years, approximately 471 patients with rheumatic mitral stenosis were treated in our centre. ${ }^{2}$ Treatments provided for RHD in advanced stages are relatively expensive for developing nations; thus, early detection and targeted treatment can greatly aid. ${ }^{3}$ Mitral valve stenosis is the main presentation of RHD, commonly developing as a result of persistent or recurrent valvulitis with bicommissural fusion. ${ }^{4}$ Fibrogenesis is induced by various stimuli, such as cytokines, connective tissue growth factors and activators. Previous studies suggest that RHD is an autoimmune disease that is associated with cytokine activation. ${ }^{4}$ Inflammatory cytokines are key regulators of immune processes. Chronic inflammation causes damage to the valvular tissue. Many studies have investigated potential biomarkers to evaluate fibrosis and chronic inflammation processes in patients with RHD and ST2 is a sensitive marker for detecting cardiac fibrosis, including fibrosis progression in RHD. ${ }^{4-6}$ 
ST2 is a member of the interleukin (IL)-1 receptor family discovered in a classical translational science fashion, and it exists in two forms: a transmembrane receptor (ST2L) and a soluble decoy receptor (sST2). ${ }^{7}$ As a member of the IL-1 receptor family, ST2 is a biomarker of mechanical stress that is upregulated in isolated cardiomyocytes exposed to mechanical strain; derangement of ST2 signalling leads to a phenotype consistent with myocardial remodelling, and in patients with heart failure, sST2 levels strongly correlate with the severity of heart failure, independently forecasting risk on top of the risk from NT-proBNP and other biomarkers. Both sST2 and ST2L are induced in cardiomyocytes and fibroblasts exposed to biomechanical stress. Biomechanical stress and fibrosis will enhance valve thickening in RHD. ${ }^{8}$ Clarifying the role played by ST2 in cardiovascular disease, IL-33 signalling through ST2L has been shown to have antihypertrophic and antifibrotic effects in the heart. ${ }^{7}$ Calcification and thickening of the mitral valves are enhanced by the production of angiotensin II. Angiotensin II induces the upregulation of transforming growth factor $\beta$ and later the binding of IL-33 to sST2 instead of its natural receptor ST2L. Binding of IL-33 to sST2 will cause fibrogenesis. Thus, ACE inhibitors (ACEIs) are hypothesised to attenuate this vicious cycle by inhibiting angiotensin II and consequently increasing bradykinin, which further inhibit fibrosis through the negative regulation of angiotensin II activity in mitogen-activated protein kinase pathways through the suppression of the Ca2 + response and $\mathrm{Na}+$ transport. $^{9} 10$

ACEIs are frequently used to prevent and treat heart failure caused by regurgitant valve disease. Because of the risk of hypotension in the presence of a fixed obstruction, the majority of patients with symptomatic RHD have substantial mitral stenosis (MS) and refuse ACEI medication. ${ }^{11}$ ACEI is the primary treatment for heart failure. The way ACEIs improve clinical symptoms and survival outcomes is to advance afterload reduction. Fibrosis attenuation and its antiproliferative effects and neurohormonal effects are superior to those of pure vasodilators. ${ }^{11}$ Current guidelines for valvular intervention do not include ACEI as therapy in patients with rheumatic MS. The only established therapeutical options for rheumatic MS are balloon mitral valvuloplasty and mitral valve surgery (MVS). More economical therapeutical options that target the inhibition of fibrogenesis and improve mitral valve fibrosis are needed, especially in low-income to middle-income countries. Valvular anti-inflammatory and anti-fibrotic medical therapy to slow the progression of the disease is needed in patients with rheumatic MS. One ACEI (enalapril) was well tolerated in symptomatic RHD associated with significant aortic and/or MS and preserved left ventricular systolic function. ${ }^{11}$

Currently, there is no treatment for rheumatic MS that targets the main pathogenesis, valvular fibrosis. Therefore, novel approaches and therapies are needed to prevent RHD progression. ${ }^{4}$ Neutralising inflammatory cytokines or antagonising their receptor function has

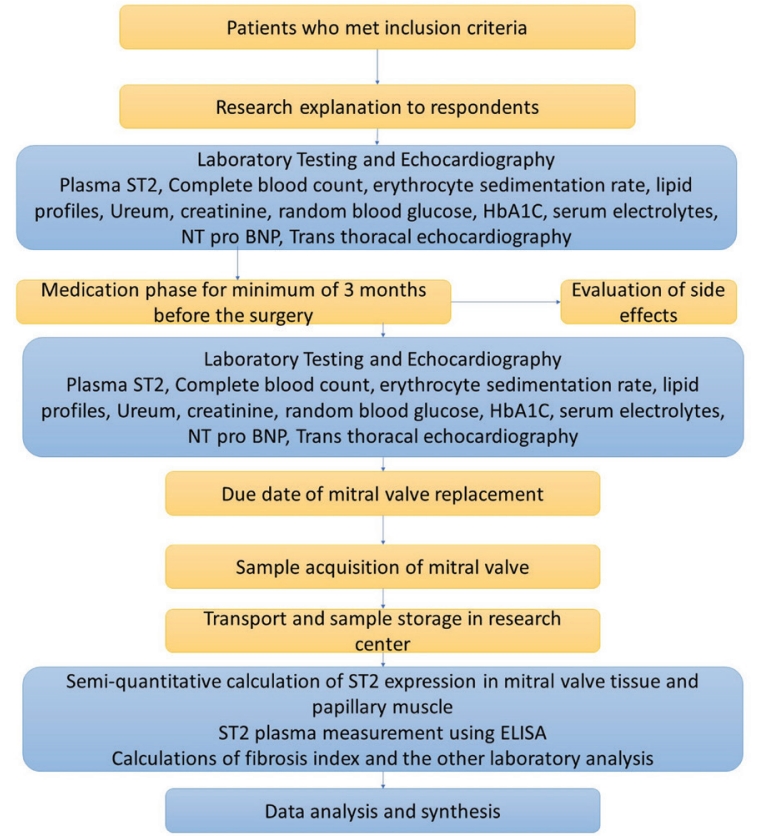

Figure 1 Hypothesis. Molecular mimicry is a defence mechanism of group A Streptococcus to avoid immune cells. This mechanism allows immune cells to generate autoimmunity against protein the lining of endothelial cells and causing chronic inflammation and valvular damage. Continuous process of chronic inflammation leads to valvular thickening and fibrosis, which is mediated by the angiotensin II. Angiotensin II increase TGF- $\beta$ expression and cause IL33 to bind with sST2, and subsequently cause damage and fibrosis to the valvular tissue even more, which later will end with rheumatic heart failure. ACEl is hypothesised to counteract these processes by decreasing angiotensin II conversion from angiotensin I. ACEI, ACE inhibitor; IL, interleukin; TGF- $\beta$, transforming growth factor $\beta$.

been considered a useful therapeutical strategy to treat autoimmune diseases. ${ }^{4}$ In this respect, new therapies targeting ST2 and its ligands, as studied in some autoimmune diseases, may be a new approach for patients with RHD. ACEIs are agents with antifibrotic effects. This study therefore aims to investigate the effect of the ACEI ramipril in suppressing the expression of ST2 in the cardiac mitral valve in patients with RHD (figure 1).

\section{METHODS AND ANALYSIS Study designs}

This is a single-centre, double-blind, placebo-controlled, randomised clinical trial with a pre-post test design. Patients with rheumatic MS with valvular dysfunction who are scheduled for cardiac valve replacement will be treated with ramipril $5 \mathrm{mg}$ or placebo for a minimum of 12 weeks (3 months) before the surgery. ST2 will be checked as a fibrosis marker (figure 2). The study is still recruiting patients at the National Cardiac Center Harapan Kita Hospital, Jakarta, Indonesia, from 27 June 2019. 


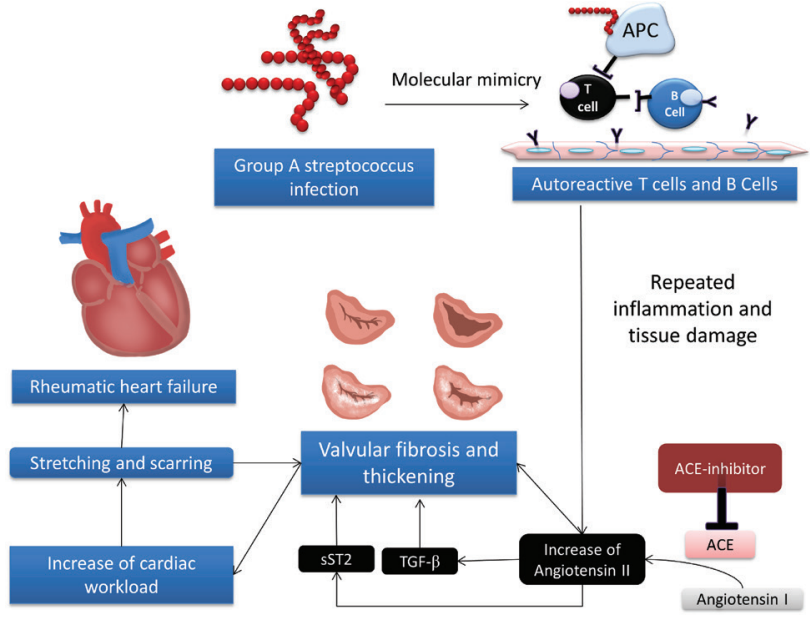

Figure 2 Research flowchart. TGF- $\beta$, transforming growth factor $\beta$.

\section{Study population}

Patients with rheumatic mitral valve stenosis (RMS) who undergo cardiac valve replacement in the National Cardiovascular Center Harapan Kita (NCCHK), Jakarta, Indonesia, will be screened for eligibility. The inclusion criteria of this study are patients with RMS or combined valve disease aged more than 18 years who undergo cardiac valve replacement operation with or without tricuspid valve repair. Patients must also have systolic blood pressure (SBP) $\geq 100 \mathrm{~mm} \mathrm{Hg}$ and diastolic blood pressure $\geq 60 \mathrm{~mm} \mathrm{Hg}$. The exclusion criteria of this study are patients with congenital heart disease, non-MVS, coronary artery bypass surgery or refusal to provide informed consent. Further exclusion criteria are adults aged 65 years or over, pregnant women and patients with autoimmune disease, persistent hypotension (SBP $<100 \mathrm{~mm}$ $\mathrm{Hg}$ ), severe aortic stenosis (aortic valve orifice $<0.75 \mathrm{~cm}^{2}$ ), chronic renal dysfunction with serum creatinine $>2.5 \mathrm{mg}$ / $\mathrm{dL}$ or known ACEI intolerance. Participants who meet the criteria and are willing to join the RamiRHeD trial will be informed in detail about the study and will be required to sign the informed consent.

\section{Outcomes}

The primary outcomes of this study are the ST2 expression in mitral valve tissue and papillary muscle, and the secondary outcomes are soluble plasma ST2, clinical signs and symptoms that will be measured with the classification of NYHA (New York Heart Association), echocardiography results of: ejection fraction, TAPSE (tricuspid annular plane systolic excursion), end diastolic dimension, end systolic dimension, mitral valve area, mitral valve gradient, tricuspid maximal velocity and tricuspid regurgitation severity, as well as laboratory test results for NT-proBNP concentration. Study participants will be followed-up for cardiac and all-cause mortality outcomes until 1 year after the surgery.

\section{Sample size and randomisation}

This is a pioneering study analysing the effects of $5 \mathrm{mg}$ ramipril on ST2 expression in mitral valve tissue in humans. A previous study that used ST2 human tissue was conducted by Marzullo et al in $2016 .{ }^{12}$ The used carotid tissue from carotid endarterectomy, with a sample size of 41 consecutive patients. Because our study will use human tissue samples, we approached the sample size calculation using the multistage non-finite population method, using this specified precision estimation formula ${ }^{13}: \mathrm{N}=(\mathrm{Z} \delta) / \mathrm{E}$, with $\mathrm{N}=$ sample size; $\mathrm{Z}_{0.95}=1.96 ; \delta \mathrm{N}(0,1)=1$; and $\mathrm{E}=0.05$ for a 0.95 CI. Therefore, we calculated a required sample of $1.65(1) / 0.05=33$ samples.

According to the sample size of the previous study that analysed ST in human tissue and a sample size formula that is commonly used in in vivo studies, we decided to use a sample size of 30 for each arm, and with the addition of a drop out rate of $10 \%$, this became total of 66 for the two arms.

The number includes a $10 \%$ dropout and withdrawal from each group. Randomisation will be done with an equal ratio of ramipril to placebo. A computerised sequence generator is used for randomisation. It will be linked with codes for placebo and treatment tablets provided by the manufacturer that was contracted to produce the trial medication. Researchers and participants will be blinded. After randomisation, the treatment pack and capsule will be identical between the two groups and will contain either active tablets or placebo. The principal investigator will have no access to the randomisation list.

\section{Research technique}

The MVS will be mitral valve replacement. Echocardiography will establish the diagnosis of rheumatic mitral valve disease. Rheumatic valve disease will be diagnosed with World Heart Federation Criteria for RHD. ${ }^{14}$ The reference measurement for valve area is planimetry by two-dimensional echocardiography. The Doppler technique is used to assess the mean mitral gradient. Seller's classification on left ventriculography in a right anterior oblique view angle of $30^{\circ}$ will be performed to evaluate the severity of mitral valve regurgitation. In cases of missing data, substitution measurements will be used as previously described: Doppler half-time pressure for valve area and colour Doppler for mitral regurgitation. ${ }^{14}$

Patient classification and diagnosis of rheumatic MS will be determined by qualified cardiologists, and the decision to perform mitral valve replacement surgery will be based on the consensus of the multidisciplinary team, consisting of cardiologists and cardiothoracic surgeons. Echocardiography will be performed by echocardiographyconsultant cardiologists. Blood samples will be collected by trained nurses specialised in pathology clinical laboratory work. Biomedical analysts will be in charge of the analysis and collection of ST2 in plasma and mitral valves. Detailed interviews with the study participants will be done by a well-trained medical doctor. The data will come 
from questionnaires, laboratory tests, echocardiography and biochemical tests. The study instruments will use the same technique, same tools, same brands and same place for data collection from each study participant.

Pre-existing atrial fibrillation, left atrial size, concomitant rheumatic valve disease, NYHA class and other clinical data and echocardiographic data will be documented before and after surgery and will be analysed by multivariate analysis.

\section{Intervention}

Daily capsules containing $5 \mathrm{mg}$ ramipril or placebo to be taken orally will be provided for the study participants. An initial dose of $2.5 \mathrm{mg}$ of ramipril will be given to the patients in the intervention group. If there are no significant adverse effects documented in the first 2 weeks after the initial dose, $5 \mathrm{mg}$ of ramipril will be given in the subsequent weeks until 5 days before mitral valve replacement surgery. Participants will remain under the care of the treating cardiologist team. The routine medications of each patient will be continued. Capsules containing $5 \mathrm{mg}$ ramipril or placebo will be given for a minimum of 3 months, up until 5 days before the mitral valve replacement.

\section{Withdrawal and drop out}

Participants will be informed that they will be able to withdraw from the study at any time and will sign a form stating this. They will be informed that this will not affect their clinical care. Basic clinical data and samples already collected will be included in the analyses in accordance with the consent obtained at trial entry. Drop-out criteria will be loss to follow-up, severe adverse events and mortality due to any cause.

\section{Sample collection and measurements}

Clinical signs and symptoms will be documented before and after the study. Blood samples will be collected twice: before the intervention and 1 day before the MVS. The routine blood analysis will include haemoglobin, platelet count, leucocyte count, erythrocyte sedimentation rate and C-reactive protein. Total cholesterol, random blood glucose, HbAlc, urea, creatinine, serum electrolytes, NT-proBNP and plasma ST2 will be determined. Echocardiography before the intervention and before surgery will be performed. Mitral valve tissue expression of ST2 will be measured by immunohistochemistry (IHC). Plasma ST2 will be measured using an ELISA kit with the human ST2/IL-33R antibody (R\&D Systems, catalogue number DST200). This assay uses the technique of the quantitative sandwich enzyme immunoassay. A monoclonal antibody specific for human ST2 is precoated onto a microplate. Standards and samples are pipetted into the wells, and any ST2 present is bound by the immobilised antibody. Unbound substances are washed away and then, an enzyme-linked polyclonal antibody specific for human ST2 is added to the wells. Following a wash to remove any unbound antibody-enzyme reagent, a substrate solution is added to the wells, and colour develops in proportion to the amount of ST2 bound in the initial step. After the colour development is stopped, the colour intensity is measured.

Mitral valve and papillary muscle tissue will be collected during mitral valve replacement surgery and will be saved in a sterile container filled with $10 \%$ formalin. ST2 expression will be observed using IHC. Cross-linking chemicals, such as paraformaldehyde and glutaraldehyde, will be used to preserve the cellular structure. The fixation begins when the tissue is harvested. Tissue blocking is performed afterwards by placing the tissue sample in hot parafilm, after which it is put into a mould until hard. Following fixation, tissue sections are obtained using a microtome. Decloaking methods consisting of heat and pressure treatment, enzyme digestion and microwaving are done afterwards. Following decloaking, the parafilm on the slides is removed by baking, and then the IHC staining process can be started. The primary antibody is a monoclonal ST2 antibody. The secondary antibody is conjugated by biotin. The blocking buffer includes BSA. The chromogen that will be used is 3,3'-diaminobenzidine $(\mathrm{DAB})$. DAB oxidation is catalysed by horseradish peroxidase, after which it forms a brown precipitate, so ST2 expression can be visualised under a light microscope. The tissue will then be counterstained using H\&E staining, so the non-ST2-expressing cells can be visualised in bluish colour. A negative control will use H\&E staining only. Measurements of cells that express ST2 will be performed under a microscope. The date, tissue type, antibody dilution, tissue treatment and magnification of the microscope will be documented. ST2-expressing cells will be counted by more than one professional.

\section{Statistical analysis}

Continuous variables are expressed as mean $\pm \mathrm{SD}$, and categorical variables are expressed as percentages. The $\chi^{2}$ test will be used to see the relationship between dichotomous variables, and Student's t-test will be used for continuous variables. Single-variable correlation analysis and multivariable linear regression analysis will be performed. A p value $<0.05$ is considered statistically significant. The analyses will be performed with SPSS (IBM Corp., Armonk, NY, USA) for Windows.

\section{Ethics and dissemination}

The ethics of this study were approved by the ethics committee of NCCHK, Jakarta, Indonesia, with ethical code LB.02.01/VII/286/KEP.009/2018.

\section{DISCUSSION}

This study is planning to recruit patients with rheumatic mitral valve to be randomised to obtain capsules containing either ramipril $5 \mathrm{mg}$ or placebo. Rheumatic MS is the main presentation of RHD that leads to significant morbidity and mortality. Recurrent or persistent valvulitis with bicommisural fusion usually leads to MS. Previous 
studies suggest that RHD is an autoimmune disease that is associated with cytokine activities. Inflammatory cytokines are key regulators of immune processes. ${ }^{4}$ Immunological reactions caused by autoreactive antibodies continuously cause chronic inflammation and valvular fibrosis, which can be detected by an increase in sST2, an emerging biomarker for cardiac fibrosis. ${ }^{101516}$

IL-33 is the natural ligand of ST2 and is highly expressed in smooth muscles and airway epithelia. ${ }^{17} \mathrm{An}$ inflammatory state stimulates the upregulation of ST2 by some cells, such as keratinocytes and dermal fibroblasts, and mechanical strain upregulates ST2 in cardiac fibroblasts. ${ }^{17} 18$ The soluble ST2 isoform is increased under inflammatory conditions such as sepsis, allergic asthma, trauma and pulmonary diseases. ${ }^{19-22}$ Its elevation is also documented in some heart conditions, such as aortic stenosis and congestive cardiomyopathy, and this elevation is associated with the risk of heart failure and death. ${ }^{23-27}$ In this study, plasma ST2 is considered an inflammatory and fibrotic biomarker of rheumatic MS. Because plasma ST2 can also increase in various conditions unrelated to cardiac fibrosis, this study also measures the ST2 expression in mitral valve tissue. Plasma ST2 describes the amount of ST2 in the circulation, whereas mitral valve cells that express ST2 describe the amount of transmembrane ST2.

ACEIs are commonly administered as the treatment of heart failure due to valvular regurgitation. Its use in MS is still debatable because of its hypotensive effect. A prior study assessing the safety of ACEIs in patients with MS showed that the ACEI enalapril was well tolerated and safe up to a dose of $10 \mathrm{mg}$ two times per day. ${ }^{11}$ ACEIs are presumed to have vasodilatory effects in obstructive lesions and will decrease systemic vascular resistance through arterial vasodilatation, thus increasing the transvalvular gradient. Their anti-remodelling effect is also well established, and their long-term use has also been proven to improve left ventricular ejection fraction in patients with systolic dysfunction. ${ }^{28}$ Because a prior study ${ }^{11}$ demonstrated the efficacy and the potential benefits of ACEIs in improving outcomes in patients with MS, this study aims to confirm and investigate the possible pathological mechanism of those improvements. This study will assess the effect of $5 \mathrm{mg}$ ramipril as a cardiac antifibrosis treatment in patients with severe MS RHD. Their plasma ST2 concentrations will be compared. Plasma ST2 concentration will also be compared before and after several months of consuming $5 \mathrm{mg}$ ramipril. There will be no healthy controls for this study because of ethical limitations in the acquisition of mitral valve tissue. Mitral valve tissue will be acquired during MVS. The expression of ST2 in mitral valve tissue will then be calculated semiquantitatively and compared with the plasma ST2 results. It is hypothesised that ramipril will suppress the expression of ST2 in the cardiac mitral valve in patients with RHD.

In addition to the plasma ST2 level and the ST2 expression in mitral valve tissue, this study also compares the pre-post effects of $5 \mathrm{mg}$ ramipril versus placebo on NT-proBNP concentration echocardiography strain parameters and clinical outcomes. Clinical signs and symptoms and echocardiography parameters have been evaluated in some studies of mitral valve stenosis, and showed that these were positively correlated with the NT-proBNP concentration. ${ }^{29}{ }^{30}$ This study will also compare the NT-proBNP concentration between patients receiving ramipril and placebo. We will also calculate the correlation between the NT-proBNP concentration and the ST2 plasma concentration and mitral valve expression.

\section{Author affiliations}

${ }^{1}$ Department of Cardiovascular Prevention and Rehabilitation, National Cardiovascular Center Harapan Kita, West Jakarta, Jakarta, Indonesia

${ }^{2}$ Department of Cardiology and Vascular Medicine, Faculty of Medicine, Universitas Indonesia, West Jakarta, Jakarta, Indonesia

${ }^{3}$ Research Assistant of Department of Cardiovascular Prevention and Rehabilitation, National Cardiovascular Center Harapan Kita, West Jakarta, Jakarta, Indonesia ${ }^{4}$ Department of Cardiology, University Medical Centre Utrecht, Utrecht, The Netherlands

${ }^{5}$ Central Military Hospital, Netherlands Heart Institute, Utrecht, The Netherlands

Contributors Conception and design of the work was initiated by AMA, BS, $\mathrm{AS}, \mathrm{BR}$ and $\mathrm{BD}$. AMA, BD, ES and FT contributed to the acquisition, analysis and interpretation of data for the work. This manuscript was drafted by AMA, BD and ES. AMA, PD, AW and MJMC critically revised the manuscript. The author and coauthors gave final approval and agree to be accountable for all aspects of the work and ensuring its integrity and accuracy.

Funding The authors have not declared a specific grant for this research from any funding agency in the public, commercial or not-for-profit sectors.

\section{Competing interests None declared.}

Patient and public involvement Patients and/or the public were not involved in the design, or conduct, or reporting, or dissemination plans of this research.

\section{Patient consent for publication Obtained.}

Provenance and peer review Not commissioned; externally peer reviewed.

Open access This is an open access article distributed in accordance with the Creative Commons Attribution Non Commercial (CC BY-NC 4.0) license, which permits others to distribute, remix, adapt, build upon this work non-commercially, and license their derivative works on different terms, provided the original work is properly cited, appropriate credit is given, any changes made indicated, and the use is non-commercial. See: http://creativecommons.org/licenses/by-nc/4.0/.

ORCID iD

Ade Meidian Ambari http://orcid.org/0000-0002-7488-4107

\section{REFERENCES}

1 Rodriguez-Fernandez R, Amiya R, Wyber R, et al. Rheumatic heart disease among adults in a mining community of Papua, Indonesia: findings from an occupational cohort. Heart Asia 2015;7:44-8.

2 Ambari AM, Setianto B, Santoso A, et al. Survival analysis of patients with rheumatic MS after PBMV compared with MVS in a low-tomiddle-income country. Neth Heart J 2019;27:559-64.

3 Sharma N, Toor D. Interleukin-10: role in increasing susceptibility and pathogenesis of rheumatic fever/rheumatic heart disease. Cytokine 2017;90:169-76.

4 Bilik MZ, Kaplan İbrahim, Polat N, et al. Serum levels of IL-17 and IL-23 in patients with rheumatic mitral stenosis. Medicine 2016;95:e3562.

5 Ramona J, Alexander von M, Martin F, et al. Soluble ST2 - A Potential Biomarker of Rheumatic Heart Disease. Clin Med Rev Case Rep 2019;6.

6 Gardezi SK, Coffey S, Prendergast BD, et al. Serum biomarkers in valvular heart disease. Heart 2018;104:349-58.

7 Januzzi JL. St2 as a cardiovascular risk biomarker: from the bench to the bedside. J Cardiovasc Trans/ Res 2013;6:493-500. 
8 Ayoub S, Ferrari G, Gorman RC, et al. Heart valve biomechanics and underlying mechanobiology. Compr Physiol 2016;6:1743-80.

9 Hus-Citharel A, Bouby N, Iturrioz X, et al. Multiple cross talk between angiotensin II, bradykinin, and insulin signaling in the cortical thick ascending limb of rat kidney. Endocrinology 2010;151:3181-94.

10 Ambari AM, Setianto B, Santoso A, et al. Angiotensin converting enzyme inhibitors (ACEls) decrease the progression of cardiac fibrosis in rheumatic heart disease through the inhibition of IL-33/ sST2. Front Cardiovasc Med 2020;7:115.

11 Chockalingam A, Venkatesan S, Dorairajan S, et al. Safety and efficacy of enalapril in multivalvular heart disease with significant mitral stenosis--SCOPE-MS. Angiology 2005;56:151-8.

12 Marzullo A, Ambrosi F, Inchingolo M, et al. ST2L transmembrane receptor expression: an immunochemical study on endarterectomy samples. PLoS One 2016;11:e0156315-12.

13 Louangrath PI. Sample size determination for Non-Finite population. SSRN Electron J 2017:1-12.

14 Reményi B, Wilson N, Steer A, et al. World Heart Federation criteria for echocardiographic diagnosis of rheumatic heart disease--an evidence-based guideline. Nat Rev Cardiol 2012;9:297-309.

15 Kakkar R, Lee RT. The IL-33/ST2 pathway: therapeutic target and novel biomarker. Nat Rev Drug Discov 2008;7:827-40.

16 Miller AM. Role of IL-33 in inflammation and disease. J Inflamm 2011;8:22.

17 Schmitz J, Owyang A, Oldham E, et al. II-33, an interleukin-1-like cytokine that signals via the IL-1 receptor-related protein ST2 and induces T helper type 2-associated cytokines. Immunity 2005;23:479-90.

18 Sanada S, Hakuno D, Higgins LJ, et al. II-33 and ST2 comprise a critical biomechanically induced and cardioprotective signaling system. J Clin Invest 2007:117:1538-49.

19 Hayakawa H, Hayakawa M, Kume A, et al. Soluble ST2 blocks interleukin-33 signaling in allergic airway inflammation. $J$ Biol Chem 2007:282:26369-80.

20 Martinez-Rumayor A, Camargo CA, Green SM, et al. Soluble ST2 plasma concentrations predict 1-year mortality in acutely dyspneic emergency department patients with pulmonary disease. Am J Clin Pathol 2008;130:578-84.

21 Becerra A, Warke RV, Xhaja K, et al. Increased activity of indoleamine 2,3-dioxygenase in serum from acutely infected dengue patients linked to gamma interferon antiviral function. J Gen Virol 2009;90:810-7.

22 Brunner M, Krenn C, Roth G, et al. Increased levels of soluble ST2 protein and IgG1 production in patients with sepsis and trauma. Intensive Care Med 2004;30:1468-73.

23 Bartunek J, Delrue L, Van Durme F, et al. Nonmyocardial production of ST2 protein in human hypertrophy and failure is related to diastolic load. J Am Coll Cardiol 2008;52:2166-74.

24 Szerafin T, Niederpold T, Mangold A, et al. Secretion of soluble ST2 - possible explanation for systemic immunosuppression after heart surgery. Thorac Cardiovasc Surg 2009;57:25-9.

25 Sabatine MS, Morrow DA, Higgins LJ, et al. Complementary roles for biomarkers of biomechanical strain ST2 and N-terminal prohormone B-type natriuretic peptide in patients with ST-elevation myocardial infarction. Circulation 2008;117:1936-44.

26 Mueller T, Dieplinger B, Gegenhuber A, et al. Increased plasma concentrations of soluble ST2 are predictive for 1-year mortality in patients with acute destabilized heart failure. Clin Chem 2008;54:752-6.

27 Shimpo M, Morrow DA, Weinberg EO, et al. Serum levels of the interleukin-1 receptor family member ST2 predict mortality and clinical outcome in acute myocardial infarction. Circulation 2004;109:2186-90

28 Jao G, Lystash J, Sane D. Angiotensin-Converting enzyme inhibitors can increase the transvalvular gradient among patients with aortic stenosis. J Am Coll Cardiol 2012;59:777.

29 Arat-Ozkan A, Kaya A, Yigit Z, et al. Serum N-terminal Pro-BNP levels correlate with symptoms and echocardiographic findings in patients with mitral stenosis. Echocardiography 2005;22:473-8.

30 Iltumur K, Karabulut A, Yokus B, et al. N-Terminal proBNP plasma levels correlate with severity of mitral stenosis. J Heart Valve Dis 2005;14:735-41. 